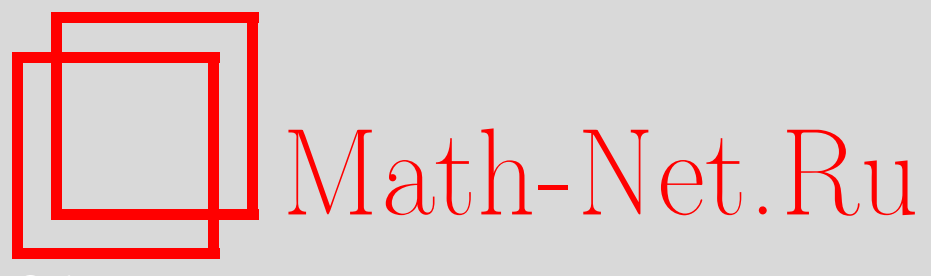

В. Д. Володин, Кубические реализации флаговых нестоэдров и доказательство гипотезы Гала для них, $У M H$, 2010, том 65, выпуск 1, 183-184

DOI: https://doi.org/10.4213/rm9327

Использование Общероссийского математического портала Math-Net.Ru подразумевает, что вы прочитали и согласны с пользовательским соглашением http://www . mathnet.ru/rus/agreement

Параметры загрузки:

IP : 3.91 .87 .62

26 апреля 2023 г., 15:37:46

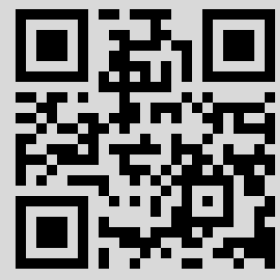




\section{Кубические реализации флаговых нестоэдров и доказательство гипотезы Гала для них}

\section{В. Д. Володин}

Для каждого $n$-мерного выпуклого многогранника $P$ можно определить $f$-полином формулой $f(P)(t)=\sum_{i=0}^{n} f_{i} t^{i}$, где $f_{i}$ - число граней размерности $i$, и $h$-полином формулой $h(P)(t)=f(P)(t-1)$. Соотношения Дена-Соммервилля для простых многогранников равносильны возвратности их $h$-полиномов, следовательно, $h(P)(t)=$ $\sum_{i=0}^{[n / 2]} \gamma_{i} t^{i}(1+t)^{n-2 i}$. Полином $\gamma(P)(\tau)=\sum_{i=0}^{[n / 2]} \gamma_{i} \tau^{i}$ называется $\gamma$-полиномом. Простой многогранник называется флаговым, если любой набор его попарно пересекающихся граней имеет непустое пересечение.

Гипотеза (Gal, [1], 2005). Если простой многогранник $P$ флаговый, mо $\gamma_{i}(P) \geqslant 0$.

ОПредЕлЕниЕ 1 . Совокупность $B$ непустых подмножеств множества $[n+1]=$ $\{1, \ldots, n+1\}$ называется производящим множеством на $[n+1]$, если: 1$)\{i\} \in B$ для всех $i \in[n+1]$; 2) $S_{1}, S_{2} \in B$ и $S_{1} \cap S_{2} \neq \varnothing \Rightarrow S_{1} \cup S_{2} \in B$. Нестоэдром $P_{B}$ производящего множества $B$ называется сумма Минковского $\sum_{S \in B} \Delta_{S} \subset \mathbb{R}^{n+1}$, где $\Delta_{S}=$ $\operatorname{conv}\left\{e_{i}, i \in S\right\} \subset \mathbb{R}^{n+1}$. Каждый нестоэдр является простым многогранником (см. [2]).

Гипотеза Гала была доказана для нестоэдров, соответствующих хордовым производящим множествам (см. [2]), и для нестоэдров, соответствующих полным двудольным графам $K_{m, n}$ (см. [3], [4]). Центральный результат этой работы: гипотеза Гала верна для всех флаговых нестоэдров.

Рассмотрим множество $\mathcal{P}^{\text {cube }}$ многогранников, получающихся из куба последовательной срезкой граней коразмерности 2. Следующий результат является двойственным к предложению 2.4.6 из [1].

ПредЛожение 2. При срезке грани коразмерности 2 простой флаговый многогранник остается простым и флаговым.

ПредЛОжение 3. Если $P \in \mathcal{P}^{\text {cube }}$, mo $\gamma_{i}(P) \geqslant 0$.

Доказательство. Пусть $P^{\prime}$ получен из $P$ срезкой грани $G \subset P$ коразмерности 2 . Тогда $\gamma\left(P^{\prime}\right)=\gamma(P)+\tau \gamma(G)$. Индукция завершает доказательство.

Согласно [5], любой нестоэдр $P_{B}$ получается срезками граней из симплекса. Здесь мы покажем, что любой флаговый нестоэдр получается срезками граней коразмерности 2 из куба. Для ассоциэдров этот факт был отмечен в [6].

Производящее множество $B$ на $[n+1]$ называется связным, если $[n+1] \in B$. В этом случае $P_{B}$ имеет размерность $n$, а его гиперграни находятся во взаимно однозначном соответствии с элементами $S$ множества $B \backslash[n+1]$ и обозначаются $F_{S}$. Решетка граней нестоэдра описывается следующим образом.

Гиперграни $F_{S_{1}}, \ldots, F_{S_{k}}$ имеют непустое пересечение тогда и только тогда, когда:

1) для любых $S_{i}, S_{j}$ или $S_{i} \subset S_{j}$, или $S_{j} \subset S_{i}$, или $S_{i} \cap S_{j}=\varnothing$;

2) для любых $S_{i_{1}}, \ldots, S_{i_{p}}$ таких, что $S_{i_{j}} \cap S_{i_{l}}=\varnothing$, выполнено $S_{i_{1}} \sqcup \cdots \sqcup S_{i_{p}} \notin B$.

Конструкция Н. Ероховца. Пусть $B, B_{1}, \ldots, B_{n+1}-$ связные производящие множества на $[n+1],\left[k_{1}\right], \ldots,\left[k_{n+1}\right]$. Тогда определено связное производящее множество $B\left(B_{1}, \ldots, B_{n+1}\right)$ на $\left[k_{1}+\cdots+k_{n+1}\right]$, состоящее из $S^{i} \in B_{i}$ и $\bigsqcup_{i \in S}\left[k_{i}\right], S \in B$.

ЛЕмма 4 (Н. Ероховец). Пусть $B, B_{1}, \ldots, B_{n+1}$ - связные производящие множества на $[n+1],\left[k_{1}\right], \ldots,\left[k_{n+1}\right]$ и $B^{\prime}=B\left(B_{1}, \ldots, B_{n+1}\right)$, тогда многогранник $P_{B^{\prime}}$ комбинаторно эквивалентен многограннику $P_{B} \times P_{B_{1}} \times \cdots \times P_{B_{n+1}}$.

Следствие 5. Каждый нестоэдр $P_{B}$ комбинаторно эквивалентен нестоэдру $P_{B_{1}}$, где производящее множество $B_{1}$ связное. 
Далее, без ограничения общности, будем рассматривать только связные производящие множества.

Разложение $S \in B_{2}$ по элементам $B_{1}$. Пусть $B_{1}$ и $B_{2}$ - связные производящие множества на $[n+1]$ и $B_{1} \subset B_{2}$. Тогда назовем набор $S_{1}, \ldots, S_{k} \in B_{1}$ разложением $S \in B_{2}$ по элементам $B_{1}$, если $S=S_{1} \sqcup \cdots \sqcup S_{k}$ и для любого $J \subset[k]$ такого, что $1<|J|<k$, верно $\bigsqcup_{j \in J} S_{j} \notin B_{1}$. Разложение существует и единственно.

Пусть $B_{1}$ и $B_{2}$ - связные производящие множества на $[n+1]$ и $B_{1} \subset B_{2}$. Занумеруем элементы $B_{2} \backslash B_{1}$ таким образом, что если $S^{i} \subseteq S^{j}$, то $i \geqslant j$. Пусть $S^{i}=S_{1}^{i} \sqcup$ $\cdots \sqcup S_{k_{i}}^{i}$ - разложение $S^{i} \in B_{2} \backslash B_{1}$ по элементам $B_{1}$. Тогда $P_{B_{2}}$ получается из $P_{B_{1}}$ последовательной срезкой граней $G^{i}=\bigcap_{j=1}^{k_{i}} F_{S_{j}^{i}}$, начиная с $i=1$. Утверждение, двойственное этому, можно найти в [5].

Лемма 6. Пусть $B_{1}$ и $B_{3}$ - связнье производящие множества на $[n+1]$ такие, что $B_{1} \subsetneq B_{3}$, а многогранники $P_{B_{1}}$ и $P_{B_{3}}-$ флаговые. Тогда найдется производящее множество $B_{2}$ такое, что $B_{1} \subsetneq B_{2} \subseteq B_{3}$, а многогранник $P_{B_{2}}$ флаговый и получается из $P_{B_{1}}$ последовательностъю срезок граней коразмерности 2.

ДокАЗАтЕЛЬСтво. Пусть $S$ - минимальный по включению элемент множества $B_{3} \backslash B_{1}$. Возьмем в качестве $B_{2}$ минимальное по включению производящее множество $\widehat{B_{1} \cup S}$, содержащее $B_{1} \cup S$. Поскольку $S \in B_{3}$, а $P_{B_{3}}$ флаговый, найдутся $I, J \in B_{3}$ такие, что $I \sqcup J=S$. Из выбора $S$ следует, что $I, J \in B_{1}$. Поэтому в разложении любого элемента $B_{2} \backslash B_{1}$ участвует не более двух множеств. Таким образом, срезаться будут только грани коразмерности 2 . Из предложения 2 следует, что $P_{B_{2}}$ флаговый.

ЛЕмма 7 (ср. [2; предложение 7.3]). Если В - связное производящее множество на $[n+1]$, а $P_{B}$ флаговый, то найдется производящее множество $B_{0} \subset B$ такое, что $P_{B_{0}}$ эквивалентно кубу $I^{n}$.

Производящему множеству, состоящему из всех подмножеств множества $[n+1]$, соответствует пермутоэдр $P e^{n}$.

СледСтвиЕ 8. Если $P_{B}$ флаговый, то последовательной срезкой граней коразмерности 2 можно получить $P_{B}$ из куба $I^{n}$ и пермутоэдр $P e^{n}$ из $P_{B}$.

Полиномы $\gamma\left(P e^{n}\right)$ удовлетворяют дифференциальному уравнению [6], из которого следует рекурсия на коэффициенты $\gamma_{i}\left(P e^{n}\right)$ и, в частности, их неотрицательность.

Теорема 9 (основной результат). 1) Пусть $P_{B}$ - флаговый нестоэдр размерности $n$, тогда $0 \leqslant \gamma_{i}\left(P_{B}\right) \leqslant \gamma_{i}\left(P e^{n}\right)$.

2) Пусть $B_{1}$ и $B_{2}$ - связные производящие множества на $[n+1]$ такие, что $B_{1} \subset B_{2}$, а $P_{B_{1}}$ и $P_{B_{2}}-$ флаговые, тогда $\gamma_{i}\left(P_{B_{1}}\right) \leqslant \gamma_{i}\left(P_{B_{2}}\right)$.

Автор благодарен В. М. Бухштаберу за постановку задачи и постоянное внимание к работе и Н. Ероховцу за ценные замечания.

\section{Список литературы}

[1] S̀. R. Gal, Discrete Comput. Geom., 34:2 (2005), 269-284. [2] A. Postnikov, V. Reiner, L. Williams, Doc. Math., 13 (2008), 207-273. [3] Н. Ю. Ероховец, Геометрия, топология и математическая физика. II, Тр. МИАН, 266, Наука, М., 2009, 127-139. [4] А. G. Fenn, arXiv: abs/0908.0605, 2009. [5] E. M. Feichtner, I. Müller, Proc. Amer. Math. Soc., 133:4 (2005), 999-1006; E.-M. Feichtner, I. Mueller, arXiv: math/0311430. [6] V. M. Buchstaber, Trends in Mathematics, 10, № 1, 2008, 1-64.

\section{В. Д. Володин (V. D. Volodin)}

Московский государственный университет им. М. В. Ломоносова

E-mail: volodinvadim@gmail.com
Представлено В. М. Бухштабером Принято редколлегией 02.11.2009 